\title{
A novel autosomal partially dominant mutation designated G476D in the keratin 5 gene causing epidermolysis bullosa simplex Weber-Cockayne type: A family study with a genetic twist
}

\author{
CEZARY KOWALEWSKI ${ }^{1}$, TAKAHIRO HAMADA ${ }^{2}$, KATARZYNA WOZNIAK ${ }^{1}$, \\ YUKO KAWANO ${ }^{2}$, WERONIKA SZCZECINSKA ${ }^{1,3}$, SHINICHIRO YASUMOTO ${ }^{2}$, \\ ROBERT A. SCHWARTZ ${ }^{4}$ and TAKASHI HASHIMOTO ${ }^{2}$ \\ ${ }^{1}$ Department of Dermatology, Medical University of Warsaw, Koszykowa 82a, 02-008 Warsaw, Poland; \\ ${ }^{2}$ Department of Dermatology, Kurume University School of Medicine, 67 Asahi-machi, Kurume-shi, \\ Fukuoka-ken 830-0011, Japan; ${ }^{3}$ Postgraduate School of Molecular Medicine, Medical University \\ of Warsaw, Warsaw, Poland; ${ }^{4}$ Department of Dermatology, New Jersey Medical School, \\ MSB H-576, 185 South Orange Avenue, Newark, New Jersey 07631, USA
}

Received August 11, 2006; Accepted January 15, 2007

\begin{abstract}
Epidermolysis bullosa simplex Weber-Cockayne type (EBS-WC) is a genetically inherited skin disease characterized by blistering restricted to the palms and soles. Its inheritance in nearly all kindreds is caused by a dominantnegative mutation in either KRT5 or KRT14, the genes encoding keratin 5 and keratin 14 proteins, respectively. Rarely, recessive mutations have also been found. We described a family with EBS-WC caused by a novel autosomal dominant mutation (G476D) in the keratin 5 gene. One family member was first seen with mucosal erosions and generalized blisters localized on the anogenital area, trunk, face and sites of mechanical trauma. Molecular analysis in this patient showed the presence of an additional mutation, an autosomal recessive $(\mathrm{G} 183 \mathrm{E})$ one, in the same gene. This observation suggests an additional effect of a recessively inherited mutation modulating the phenotypic expression of EBS caused by a partially dominant mutation and is important for accurate genetic counseling.
\end{abstract}

\section{Introduction}

Epidermolysis bullosa simplex (EBS) is a heterogenous group of genetically inherited skin diseases characterized

Correspondence to: Dr Cezary Kowalewski, Department of Dermatology, Medical University of Warsaw, Koszykowa 82a, 02008 Warsaw, Poland

E-mail: ckowalew@amwaw.edu.pl

Abbreviation: EBS, epidermolysis bullosa simplex

Key words: epidermolysis bullosa, keratin 5, electron microscopy, mutational analysis, immunofluorescence mapping by blistering of the skin following mechanical trauma. Clinically, EBS is divided into severe generalized forms and mild localized ones, most notably one variant with blistering restricted to the palms and soles (Weber-Cockayne type; EBS-WC) (1). The inheritance in nearly all kindreds is caused by a dominant-negative mutation in either gene, KRT5 encoding keratin 5 or KRT14 encoding keratin 14 proteins $(2,3)$. Rarely, recessive autosomal inheritance has also been described (4-6).

The signs and symptoms of the disease in patients of the same family are similar. Moreover, the same mutation causes similar phenotypic expression in different families. Interestingly, in a few reported EBS patients the blistering disease was more severe than in other affected family members. This difference was found to be associated with the presence of additional mutations. For example, a child of consanguineous parents with homozygous mutation M119I in the KTR 14 gene had generalized EBS, whereas heterozygotes demonstrated localized EBS-WC. Partial dominance of this mutation was suggested (7).

The mutation E170K in the gene KTR5, which causes a mild form of EBS-WC, seems to be also partially dominant. One of the reported patients with the dominant heterozygous mutation E170K was also a carrier of a recessive heterozygous mutation in the stutter region of KRT5 (8). This patient with a compound dominant-recessive heterozygous mutation had a generalized form of EBS, while his father with the heterozygous mutation E170K had blisters restricted to the soles after intensive walking. Experimental studies on MDCK cells with cDNA-transfected keratin 5 genes revealed significant keratin aggregation associated with this compound dominantrecessive heterozygous mutation.

In contrast to the partially dominant mutations responsible for the EBS-WC phenotype, a family with generalized EBS caused by the fully dominant missense 
mutation designated as $\mathrm{K} 173 \mathrm{~N}$ in region $1 \mathrm{~A}$ of the keratin 5 gene was described (9). This homozygosity for the mutant allele had clinical symptoms no more severe than that in heterozygotes. In addition, electron microscopic study did not reveal a significant difference in keratin filament formation between homozygotes and heterozygotes.

\section{Materials and methods}

Description of the family. The proband was a 14-year-old girl born at term, from non-consanguineous parents (Fig. 1). She had widespread skin fragility and generalized blistering from birth. At the time of consultation at the age of 14 years, she had mucosal erosions and generalized blisters localized on the anogenital area, trunk, face and the sites of mechanical trauma, especially on the palms and soles. Blisters on the feet were more severe during the summer than in the winter. On the face she had been constantly developing vesicles and bullae followed by erosions (Fig. 2). Lesions healed without scarring or milia formation. There was neither palmoplantar hyperkeratosis nor nail dystrophy. Skin fragility and blistering had improved with age. Her father and many of his relatives since adolescence had developed a few blisters restricted to the soles after extensive walking. The proband's mother and her relatives were unaffected.

\section{Laboratory investigations}

Immunofluorescence mapping study. To test the level of blister formation a biopsy specimen from the proband's perilesional skin was taken and immunohistochemical studies were performed using monoclonal antibodies against collagen IV (clone COL-94, Sigma) and B4 integrin (Chemicon, Temeculla, CA, USA) according to the method previously described (1).

Mutational analysis. To perform the mutational analysis, genomic DNA was extracted from blood samples obtained from the patient, her healthy mother and affected father using the protocol as previously described (10).

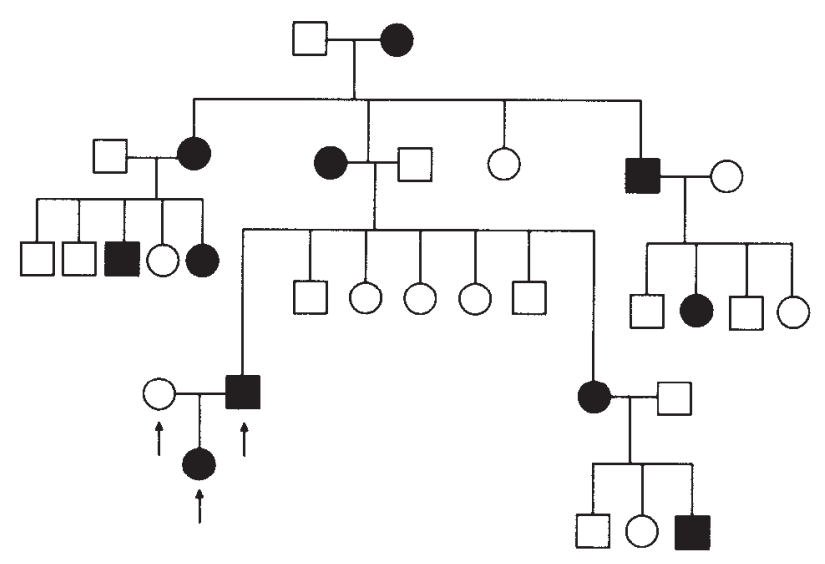

Figure 1. Pedigree of the family indicated autosomal dominant inheritance. Open symbols indicate unaffected and black symbols affected individuals. Arrows indicate the individuals from whom DNA was examined in this study (a 14-year-old girl with generalized EBS, her father suffering from EBS-WC and the healthy mother).

Electron microscopic study. Punch biopsy specimens from the involved and uninvolved skin of the proband were taken under local anesthesia, fixed in $2 \%$ glutaraldehyde and $1 \%$ osmium tetroxide, dehydrated with graded alcohol, substituted and embedded in epon. After polymerization, ultra-thin sections were cut, stained with uranyl acetate and lead citrate according to the method previously described (11) and observed under transmission electron microscopy (JEOL, model 1200EX).

\section{Results}

Immunofluorescence mapping study. The immunofluorescence mapping study confirmed the diagnosis of EBS on a biopsy specimen from the proband's perilesional skin showing the presence of collagen IV (a marker of the lamina densa) and $B 4$ integrin (a marker of the upper part of the lamina lucida) within the blister floor. Moreover, traces of
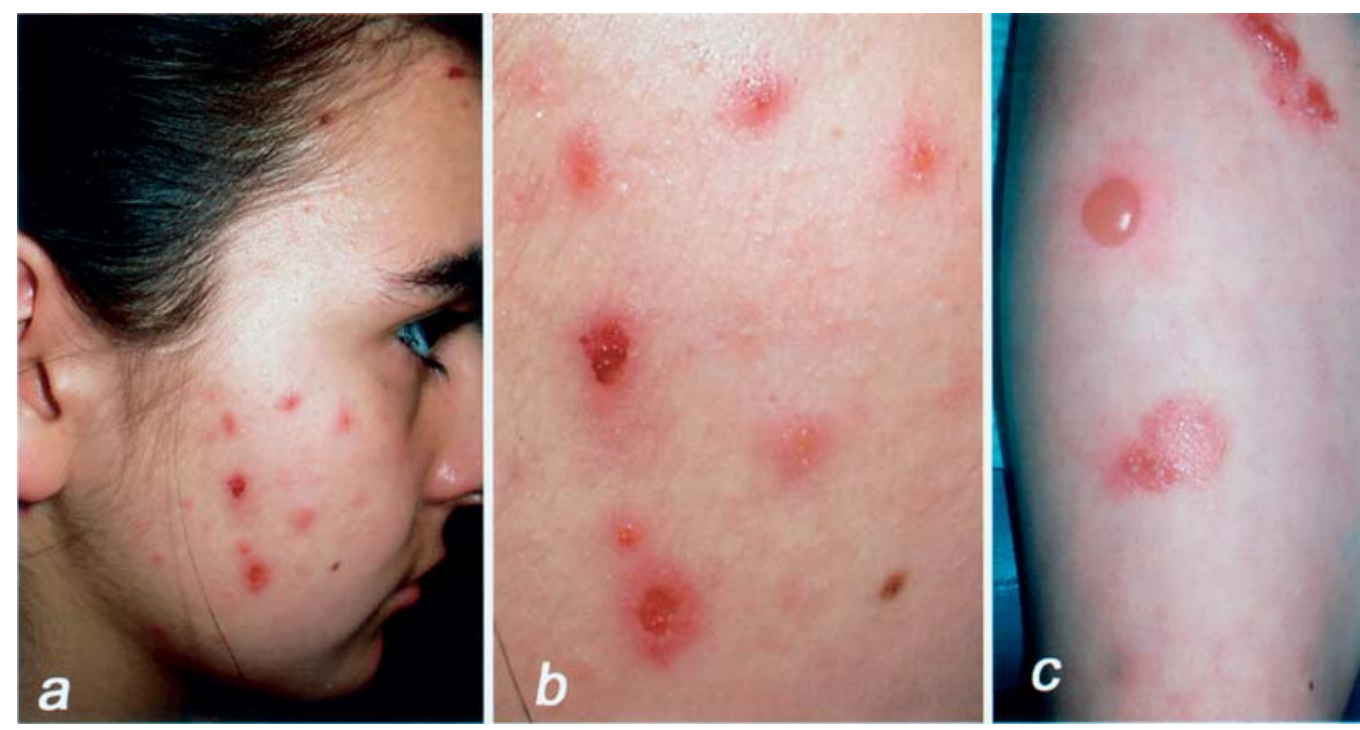

Figure 2. Clinical features of a 14-year-old patient: (a) vesicles and erosions on the face, (b) close-up of the face and (c) bulla and erosions on the thigh. 
region $1 A$, exon 1, KRT5

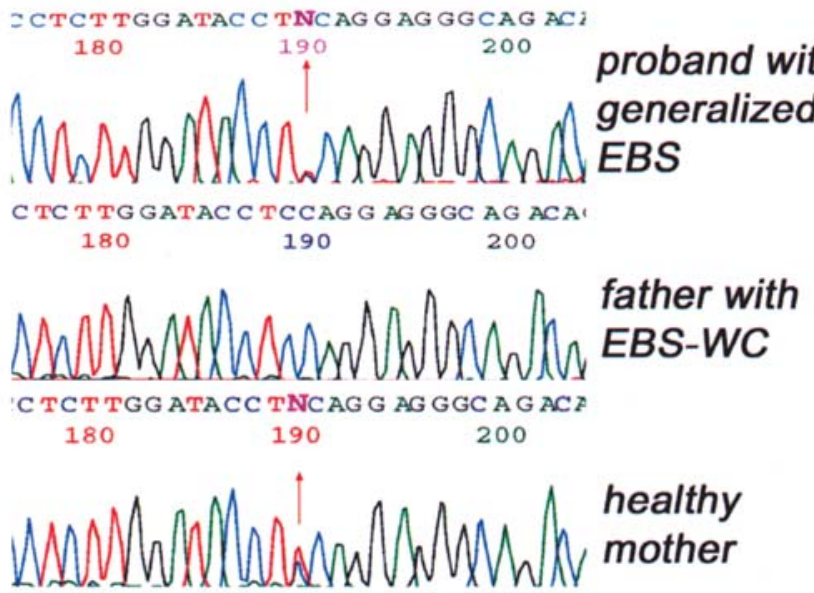

region $2 B$, exon $7, K R T 5$

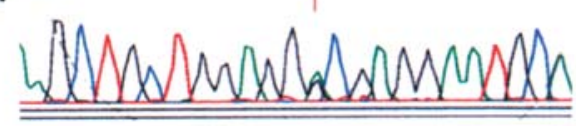

CAAGC TGC TGG AGG NCGAGG AA TGC: $250 \quad 260 \uparrow \quad 270$

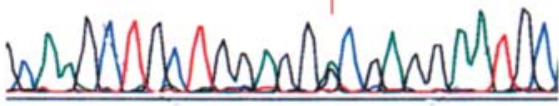

AAGC TGC TGG AGGGC GAGG AATGC $250 \quad 260$

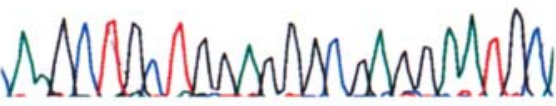

Figure 3. Direct sequencing of exon 1 of the KRT5 gene in proband with generalized EBS and her healthy mother revealed a G>A transition at nucleotide 548 that converts a glycine residue (GGA) to a glutamic acid residue (GAA); this mutation is designated G183E. The father was sequence identical with wild-type for comparison. Direct sequencing of exon 7 of the KRT5 gene in the proband with generalized EBS and her father revealed a G>A transition at nucleotide

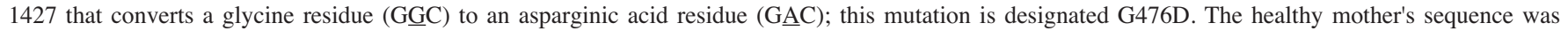
identical with wild-type for comparison.
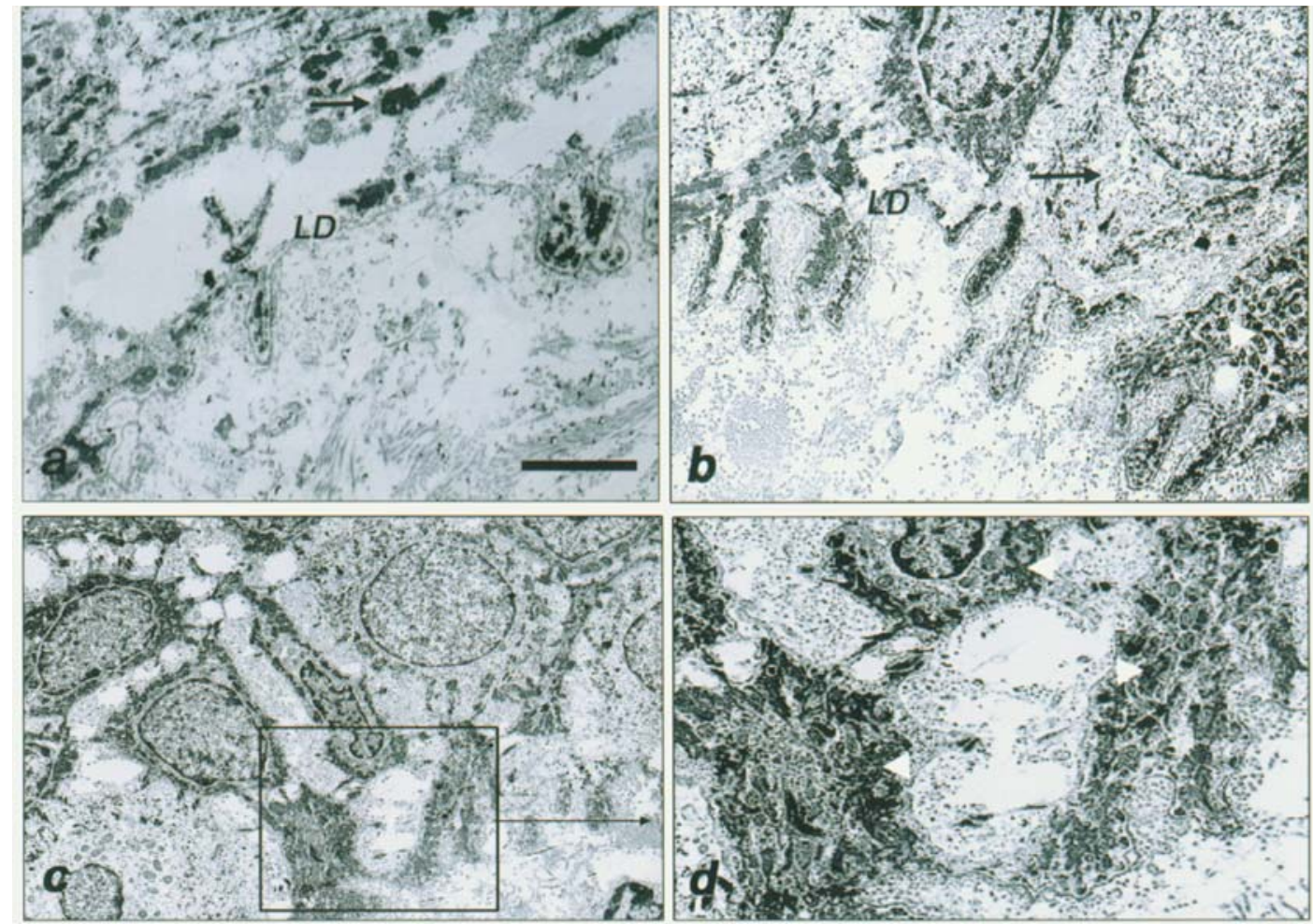

Figure 4. Electron microscopic study of biopsy specimens of the patient with generalized EBS showing: (a) intraepidermal blister and clumping of keratin filaments (arrow) in lesional skin; (b,c) gross alteration of keratin filament assembly including the reduction in the number of keratin filaments in some keratinocytes (arrow) and aggregation of keratin filaments in the others (arrowheads) in uninvolved skin; and (d) magnification x2 of marked area on c. LD, lamina densa; Bar, $1 \mu \mathrm{m}$ for $\mathrm{a}, \mathrm{b}$ and $\mathrm{c}$.

disrupted basal keratinocytes were also found on the floor of the bulla characteristic for intraepidermal separation.

Mutational analysis. Mutational analysis revealed that the proband with generalized EBS and her father with EBS-WC had a heterozygous missense mutation in exon 7 of the $2 \mathrm{~B}$ domain in the KRT5 gene changing a $\mathrm{G}>\mathrm{A}$ transition at the nucleotide at base 1427 that converts a glycine residue

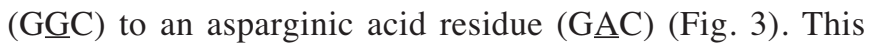
mutation is designated as G476D. Interestingly, mutational analysis of maternal genomic DNA showed the presence of a heterozygous silent mutation in region $1 \mathrm{~A}$ of the keratin 5 gene. It was a $\mathrm{G}>\mathrm{A}$ transition at nucleotide 548 that converts

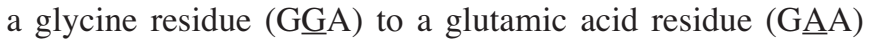
(Fig. 3). This mutation is designated as G183E and was also found in the proband. Thus, she appeared to be a carrier of a 
compound dominant-recessive heterozygous mutation. These sequence variants G476D and G183E were not found in any of the 100 control chromosomes sequenced.

Electron microscopic study. Electron microscopic study on a biopsy specimen performed from the lesional skin of the patient with generalized EBS showed the presence of an intraepidermal blister and clumping of keratin filaments (Fig. 4a).

The ultrastructural study on a biopsy specimen from the proband's uninvolved skin disclosed gross alteration of keratin filament assembly including the reduction of the number of keratin filaments in some keratinocytes and aggregation of keratin filaments in others (Fig. 4b, c and d).

\section{Discussion}

The clinical findings and disease course in the affected parent and his many relatives were consistent with the diagnosis of EBS-WC, whereas the 14-year-old proband represented a generalized form of EBS (Koebner type). The diagnosis of EBS was confirmed by immunofluorescence mapping study.

The missense mutation in the KRT5 gene designated as G476D found in the proband and her affected father is a novel mutation. Since this mutation was not found in her unaffected mother, we consider it to be a pathogenic mutation responsible for the EBS-WC phenotype. In general, the mutations associated with the mild phenotype (EBS-WC) are found in the non-helical head or linker domains $(12,13)$, whereas in generalized EBS the mutations reside in the distal end $2 \mathrm{~B}$ (or $1 \mathrm{~A}$ ) domain of the keratin 5 gene, the so-called helix initiation or termination motif (14). However, in a separate study we detected an additional two novel missense mutations in the keratin 5 and 14 genes residing in the helix initiation or termination motif in Polish families with the EBS-WC phenotype (10). Thus, the severity of EBS is associated not only with a functional site of the mutation, but also with the nature of the amino acid change and its size and structure (15).

Intriguingly, an additional missense mutation in region 1A of keratin 5 designated as G183E detected in the proband and also in her healthy mother seemed to be clinically silent when combined with the wild-type allele. However, the same mutation exacerbates the clinical severity of EBS combined with the G476D mutation on the other allele. To confirm this hypothesis, the ultrastructural organization of the keratin intermediate filaments of the proband was studied. Electron microscopic analysis of perilesional skin of the 14-year-old proband revealed gross alteration in the organization of the cytoskeleton of the basal keratinocytes including keratin aggregation and clumping and reduction in the number of intermediate filaments (Fig. 4).

In summary our study showed that an unusual course of EBS in our patient, more severe than in other family members, may have been the result of the presence of compound dominant-recessive heterozygous mutations. This observation suggests the additional effect of recessively inherited mutations modulating the phenotypic expression of EBS caused by partially dominant mutations. This finding may be important for accurate diagnosis and genetic counseling.

\section{Acknowledgements}

This study was supported by a grant from the Polish Scientific Research Committee (no. 2 PO5B 176 29), a grantin-aid for Scientific Research from the Ministry of Education, Science and Culture of Japan, a grant from the Ministry of Health and Welfare of Japan, and an Open Research Center Project grant.

\section{References}

1. Fine JD, Eady RA, Bauer EA, et al: Revised classification system for inherited epidermolysis bullosa: Report of the Second International Consensus Meeting on diagnosis and classification of epidermolysis bullosa. J Am Acad Dermatol 42: 1051-1066, 2000.

2. Stephens K, Ehrlich P, Weaver M, Le R, Spencer A and Sybert VP: Primers for exon-specific amplification of the KRT5 gene: identification of novel and recurrent mutation in epidermolysis bullosa simplex patients. J Invest Dermatol 108: 349-353, 1997.

3. Hut PH, van der Vlies P, Jonkman MF, Verlind E, Shimizu H, Buys $\mathrm{CH}$ and Scheffer H: Exempting homologous pseudogene sequences from polymerase chain reaction amplification allows genomic keratin 14 hotspot mutation analysis. J Invest Dermatol 114: 616-619, 2000.

4. Hovnanian A, Pollack E, Hilal L, Rochat A, Prost C, Barrandon Y and Goossens $\mathrm{M}$ : A missense mutation in the rod domain of keratin 14 associated with recessive epidermolysis bullosa simplex. Nat Genet 3: 327-332, 1993.

5. Jonkman MF, Heeres K, Pas HH, et al: Effect of keratin 14 ablation on the clinical and cellular phenotype in a kindred with recessive epidermolysis bullosa simplex. J Invest Dermatol 107: 764-769, 1996.

6. Indelman M, Bergman $\mathrm{R}$ and Sprecher E: A novel recessive missense mutation in KRT14 reveals striking phenotypic heterogeneity in epidermolysis bullosa simplex. J Invest Dermatol 124: 272-274, 2005.

7. Hu ZL, Smith L, Martins S, Bonifas JM, Chen H and Epstein EH Jr: Partial dominance of a keratin 14 mutation in epidermolysis bullosa simplex - increased severity of disease in a homozygote. J Invest Dermatol 109: 360-364, 1997.

8. Yasukawa K, Sawamura D, McMillan JR, Nakamura H and Shimizu H: Dominant and recessive compound heterozygous mutations in epidermolysis bullosa simplex demonstrate the role of the stutter region in keratin intermediate filament assembly. $\mathrm{J}$ Biol Chem 277: 23670-23674, 2002.

9. Stephens K, Zlotogorski A, Smith L, Erlich P, Wijsman E, Lingston RJ and Sybert VP: Epidermolysis bullosa simplex: a keratin 5 mutation is a fully dominant allele in epidermal cytoskeleton function. Am J Hum Genet 56: 577-585, 1995.

10. Hamada T, Kawano Y, Szczecinska W, Wozniak K, Yasumoto S, Kowalewski $\mathrm{C}$ and Hashimoto T: Novel keratin 5 and 14 mutations in patients with epidermolysis bullosa simplex from Poland. Arch Dermatol Res 296: 577-579, 2005.

11. Ishida-Yamamoto A, McGrath JA, Chapman SJ, Leigh IM, Lane EB and Eady RA: Epidermolysis bullosa simplex (Dowling-Meara type) is a genetic disease characterized by abnormal keratin-filament network involving keratins K5 and K14. J Invest Dermatol 97: 959-968, 1991.

12. Chan YM, Yu QC, Fine JD and Fuchs E: The genetic basis of Weber-Cockayne epidermolysis bullosa simplex. Proc Natl Acad Sci USA 90: 7414-7418, 1993.

13. Chan YM, Yu QC, LeBlanc-Straceski J, et al: Mutations in the non-helical linker segment L1-2 of keratin 5 in patients with Weber-Cockayne epidermolysis bullosa simplex. J Cell Sci 107: 765-774, 1994.

14. Corden LD and McLean WH: Human keratin diseases: hereditary fragility of specific epithelial tissues. Exp Dermatol 5: 297-307, 1996.

15. Liovic M, Stojan J, Bowden PE, Gibbs D, Vahlquist A, Lane EB and Komel R: A novel keratin 5 mutation (K5V186L) in a family with EBS-K: a conservative substitution can lead to development of different disease phenotypes. J Invest Dermatol 116: 964-969, 2001. 